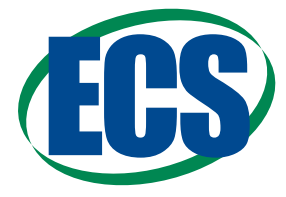

Focus Issue of Selected Papers from IMLB 2016 with Invited Papers Celebrating 25 Years of Lithium lon Batieries

\title{
Enhanced Imaging of Lithium Ion Battery Electrode Materials
}

\author{
Moshiel Biton, ${ }^{\text {a,z }}$ Vladimir Yufit, ${ }^{a}$ Farid Tariq, ${ }^{a}$ Masashi Kishimoto, ${ }^{b}$ and Nigel Brandon ${ }^{a}$ \\ ${ }^{a}$ Department of Earth Science and Engineering, Imperial College London, London, United Kingdom \\ ${ }^{b}$ Department of Aeronautics, Kyoto University, Kyoto, Japan
}

\begin{abstract}
In this study we present a novel method of lithium ion battery electrode sample preparation with a new type of epoxy impregnation, brominated $(\mathrm{Br})$ epoxy, which is introduced here for the first time for this purpose and found suitable for focused ion beam scanning electron microscope (FIB-SEM) tomography. The Br epoxy improves image contrast, which enables higher FIB-SEM resolution (3D imaging), which is amongst the highest ever reported for composite LFP cathodes using FIB-SEM. In turn it means that the particles are well defined and the size distribution of each phase can be analyzed accurately from the complex $3 \mathrm{D}$ electrode microstructure using advanced quantification algorithms.

The authors present for the first time a new methodology of contrast enhancement for 3D imaging, including novel advanced quantification, on a commercial Lithium Iron Phosphate (LFP) $\mathrm{LiFePO}_{4}$ cathode. The aim of this work is to improve the quality of the $3 \mathrm{D}$ imaging of challenging battery materials by developing methods to increase contrast between otherwise previously poorly differentiated phases. This is necessary to enable capture of the real geometry of electrode microstructures, which allows measurement of a wide range of microstructural properties such as pore/particle size distributions, surface area, tortuosity and porosity. These properties play vital roles in determining the performance of battery electrodes.

(C) The Author(s) 2016. Published by ECS. This is an open access article distributed under the terms of the Creative Commons Attribution 4.0 License (CC BY, http://creativecommons.org/licenses/by/4.0/), which permits unrestricted reuse of the work in any medium, provided the original work is properly cited. [DOI: 10.1149/2.0061701jes] All rights reserved.
\end{abstract}

Manuscript submitted June 16, 2016; revised manuscript received August 22, 2016. Published September 2, 2016. This was Paper 963 presented at the Chicago, Illinois, Meeting of the IMLB, June 19-24, 2016. This paper is part of the Focus Issue of Selected Papers from IMLB 2016 with Invited Papers Celebrating 25 Years of Lithium Ion Batteries.

In order to improve battery performance it is important to characterize and quantify real electrode microstructures in three dimensions, and understand how these structures affect performance and cycle life. ${ }^{1} \mathrm{X}$-ray and FIB-SEM techniques have been attracting a lot of interest in the field of batteries and fuel cells due to their ability to characterize electrode structure at the length scales relevant for their operation. ${ }^{2-5}$ Porous carbon electrodes play an important role in both battery and fuel cell operation, where parameters such as porosity, tortuosity and pore-size distribution determine the transport properties of the electrode, as well as the kinetics of the electrochemical reactions. ${ }^{6}$ Therefore, a thorough quantitative microstructural characterization of porous-carbon electrodes is a pre-requisite for a complete understanding of the electrode behavior. However, the quantitative 3D microstructural characterization of the porous-carbon electrodes commonly used in battery and fuel cell devices is challenging. One of the biggest difficulties in the 3D imaging of battery materials in the characterization of carbon due to (i) the low X-ray attenuation coefficient when using X-ray imaging and (ii) the non-uniform interaction with the ion beam when using FIB-SEM imaging. ${ }^{1}$

Lithium Iron Phosphate (LFP) $\mathrm{LiFePO}_{4}$ is a commercially available electrode material for Li-ion batteries for electric vehicles and energy storage applications, ${ }^{7,8}$ and was used as the basis for this study. This interest is motivated by the many advantages of this material, which include excellent cycle life and a high intrinsic safety. ${ }^{9}$ It is, however, a very challenging material to image in three dimensions since it is a porous electrode, which consists of at least three phases: LFP particles, polymer binder and conductive carbon powder, distributed at tens of nanometer length scale, which requires high resolution techniques to image carbon materials. This electrode composite emphasizes the importance of the relationship between electrode microstructure and performance in lithium ion batteries. For example, as the electronic conductivity of LFP is low ${ }^{10}$ the distribution of electronically conducting carbon black, which offsets this issue, becomes of particular importance. ${ }^{11-13}$

Figure 1 shows an SEM plan view image of an LFP electrode, a porous electrode which exhibits a variety of particle sizes, shapes and contrast highlighting the presence of both carbon particles and the LFP active material particles. For illustrative purpose, selected grains

${ }^{\text {z} E-m a i l: ~ m . b i t o n 13 @ i m p e r i a l . a c . u k ~}$ of LFP are shown in red, while some of the smaller carbon black particles are shown in green. Cooper et al. were amongst the first to study the 3D microstructure of an LFP electrode using synchrotron $\mathrm{X}$-ray microscopy. ${ }^{16}$ They pointed out that a high resolution (typically $<100 \mathrm{~nm}$ ) is required to accurately capture the LFP particle structure, which could be easily achieved using FIB-SEM techniques, as long as this method can provide sufficient contrast to distinguish between the active material, binder/carbon black and pore phases. ${ }^{16}$ The first micro CT of a cylindrical Li-ion battery cathode (Bruker high-resolution micro-CT Skyscan 1172 scanner), ${ }^{17}$ including charge/discharge cycles, and indicated that porosity and phase fraction changed as a function of ageing. Structural breakdown of the composite cathode material, such as a loss of three phase boundaries between LFP, carbon and electrolyte, and a changes in the porosity and LFP phase fraction during charge and discharge cycling, was proposed as a possible mechanism to rationalize those results. ${ }^{17}$

This work presents the next step forward in 3D reconstructing commercial battery electrodes (LFP cathode) utilizing new contrast

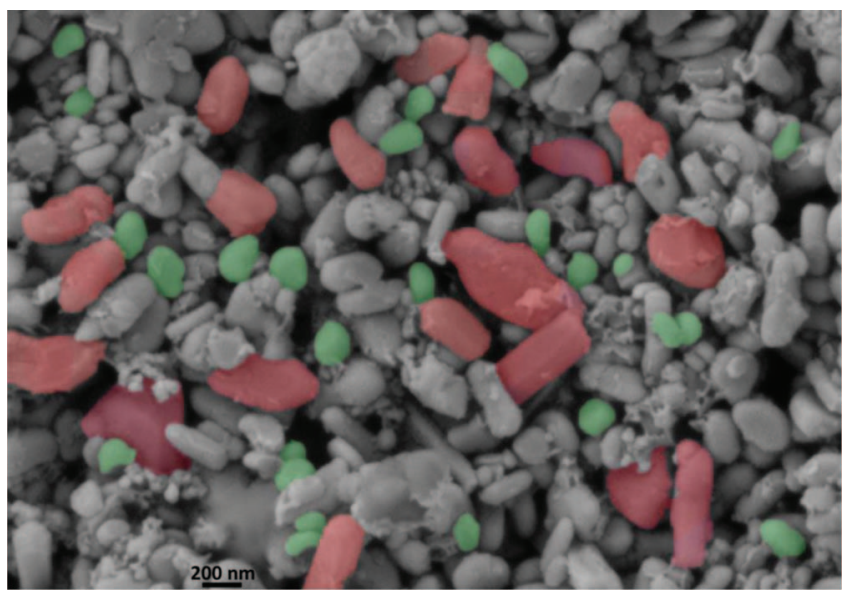

Figure 1. SEM secondary electron images of LFP electrode plan view, the selected red grains represent LFP particles while selected carbon black particles are shown in green. ${ }^{14,15}$ 
enhancing epoxies to deliver high resolution and high quality data of the three phases involved, namely the LFP active material, carbon black and pore phase; enabling each phase to be accurately analyzed from the complex 3D electrode microstructure using an advanced quantification algorithm. Though the main focus of the work here is FIB-SEM based analysis, a similar approach may be applied for X-ray tomography (see supplementary material).

In FIB-SEM tomography, a 3D reconstruction of the porous material is obtained by successive FIB milling followed by SEM imaging. However, a major drawback of this method is the lack of image contrast between solid particles and the pore space of porous materials such as the LFP electrode (Figure 1). A correct segmentation is vital for the quantification of morphology and transport parameters, which can then be used to better understand the studied material, and to develop improved materials. ${ }^{18}$ Tomographic data sets can be segmented automatically, but when there is a lack of sufficient gray level contrast a tedious manual segmentation is needed which can be done using specialized software such as Avizo (FEI, Bordeaux, France). ${ }^{19}$ Manual segmentation is time consuming and depends on the skill and experience of the operator. Hence, the use of automatic segmentation when possible is preferred. However, the common automated segmentation by relatively simple thresholding allocates pixels to a phase only according to their greyscale values, and is not capable of distinguishing between particles at the front of the pore from particles in the image background. The most direct approach to overcoming the segmentation challenge is the use of a filling material in order to obtain a contrast between pores and solid particles that enables accurate automated segmentation. Finding a filling material that gives good discrimination between pores and solid materials is not an easy task; the major requirement is that the filling material must show a strong contrast with the porous material in the SEM. As the SEM contrast can strongly depend on the atomic number, ${ }^{20}$ the filling material must consist of elements that are significantly heavier or lighter than the elements of the porous material. This is especially difficult when the sample of interest is a composite and even more so when the sample contains carbon additives since the carbon has nearly the same contrast as the commonly used epoxies, and hence cannot be differentiated in the segmentation process.

The first report of a doped epoxy pore-filling for the microstructural analysis of a fuel cell catalyst layer was reported recently. ${ }^{21}$ The report shows that the application of an epoxy doped with metal containing particles provided a smooth cross section of the layer using FIB-SEM, and provided clear bi-segmented images with bimodal grey-scale distributions. ${ }^{21}$ Another recent approach to fill the pores of the catalyst layer with $\mathrm{ZnO}$ via atomic layer deposition (ALD) prior to tomography was reported recently. ${ }^{18}$ By using ALD, even the nanometer sized pores could be filled with $\mathrm{ZnO}$, which exhibits a good contrast to the catalyst layer in SEM images. ${ }^{18}$ Of these approaches, epoxy impregnation offers the simplest approach to improve image contrast, and does not require sophisticated techniques. ${ }^{18,22}$ Hence this was the approach used in this work.

Three different types of epoxy were used for vacuum impregnation of an LFP cathode to try to improve the image contrast, and hence improve our ability to distinguish between different phases. Two of the epoxies, a carbon epoxy resin (C-epoxy) and $\mathrm{Si}$ resin (Si-epoxy), have previously been used and reported in the literature ${ }^{15,23}$ for the impregnation of LFP electrodes. Although some progress in contrast improvement was shown, artefacts and charging remained a problem and further improvement in the image quality was needed. Hence a novel (denser) brominated epoxy (Br-epoxy), never previously reported for electrode impregnation, and image contrast enhancing, is introduced in this work and compared with the results obtained using the two previously reported epoxies.

\section{Experimental}

A commercially available LFP cathode, manufactured by Hydro Quebec, was used as the basis for the study (Figure 1). The sample preparation process for imaging by FIB-SEM tomography followed
Bisphenol A diglycidyl ether, brominated

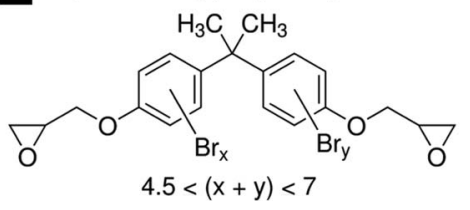

b 3-(Diethylamino) propylamine

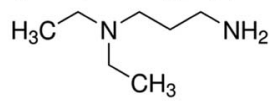

Figure 2. Chemical structures of (a) epoxy resin (Brominated -BADE) and (b) curing agent (DAPA) used for pore-filling of LFP electrode. ${ }^{25}$

that used in previous studies. ${ }^{23}$ A $3 \mathrm{~mm} \times 3 \mathrm{~mm}$ section of the electrode sheet was cut, embedded in an epoxy before being vacuum impregnated, sectioned again, attached with carbon tape and mounted onto a dedicated SEM specimen stub (Agar) using silver paste (Agar) and then coated with gold prior to FIB-SEM imaging.

The sample was characterized using SEM to capture the electrode microstructure and the shape, size and distribution of the particles. The FIB-SEM system, NVision 40, was equipped with a Gemini FE-SEM column (Carl Zeiss, Oberkochen, Germany), a zeta FIB column (SIINT, Tokyo, Japan) and a multichannel gas injection system (SIINT). An in-lens secondary electron detector was used for the microstructural imaging with an acceleration voltage around $1.5 \mathrm{kV}$. The impregnation quality and the contrast enactment were investigated. 3D imaging of the LFP electrode was conducted using FIB-SEM tomography, which sections an exposed surface of a specimen using a $\mathrm{Ga}^{+}$ion beam prior to imaging the surface with a high resolution electron beam. The process is sequentially repeated at regularly fine spaced intervals until a 3D volume size has been acquired for analysis, as detailed in several studies. ${ }^{23,24} \mathrm{~A}$ final volume of $\sim 10 \mu \mathrm{m} \times 4 \mu \mathrm{m}$ $\times 1 \mu \mathrm{m}$ with a pixel size of $10 \mathrm{~nm}$ and z-depth spacing of $15 \mathrm{~nm}$ was produced. In order to improve the segmentation process and the ability to distinguish between the phases, the porous electrode was vacuum-impregnated with three types of epoxy:

1. A C-epoxy resin SpeciFix-20 (Struers, Copenhagen, Denmark), extensively used for impregnation.

2. A Si-epoxy resin ELASTOSIL RT426 catalyzed by T-40 (Wacker Chemie AG) which was first used by Ender et al. ${ }^{12}$

3. A novel Br-epoxy. The Br-epoxy was produced by the reaction of Brominated Bisphenol A diglycidyl ether (Sigma Aldrich, St. Louis, Missouri, United States) and 3-Diethylamino propylamine (Sigma Aldrich, St. Louis, Missouri, United States), referred to as Br-BADE and DAPA respectively in the following. Their chemical structure is described in Figure $2.7 \mathrm{~g}$ of BADE were heated above its melting point to $200^{\circ} \mathrm{C}$. After $5 \mathrm{~min}$ at this temperature, $2 \mathrm{ml}$ of DAPA was added and mixed for $5 \mathrm{~min}$ and then poured on the sample and vacuum impregnated.

The resulting samples were compared in terms of contrast, impregnation quality and phase identification.

The infiltrated LFP cathode samples with $\mathrm{Si}$-epoxy and with $\mathrm{Br}$ epoxy were then encapsulated in C-epoxy to provide additional mechanical stability.

A fully automated segmentation process was carried out using only a gray level thresholding of the Avizo Fire software (FEI, Bordeaux, France). The alignment of the SEM images followed by median noise filtering to equalize the greyscale was done using Avizo. The 3D porous microstructure was reconstructed, from which microstructural parameters were quantified by QuIQ 3D software (IQM Elements, London, UK).

\section{Results and Discussion}

The sample shown in Figure 1 was impregnated with each of the three different epoxies described in the Experimental section. The quality of the impregnation and the ability to efficiently resolve different phases were the basis for comparison among the different types of epoxy. 


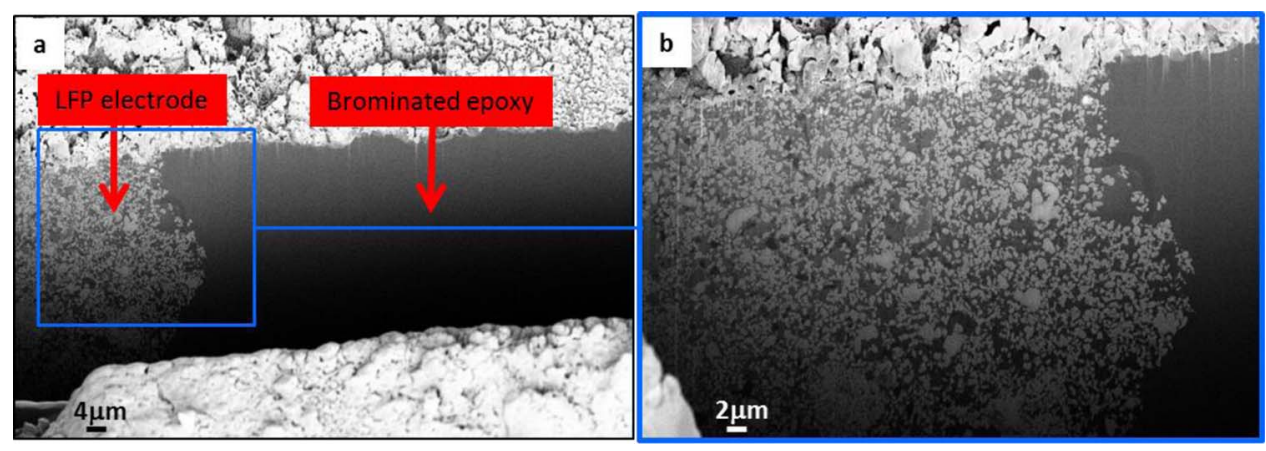

Figure 3. SEM micrographs emphasising the impregnation quality of the Br-epoxy as one homogenous phase (a) and (b) a magnified image of a region of interest.

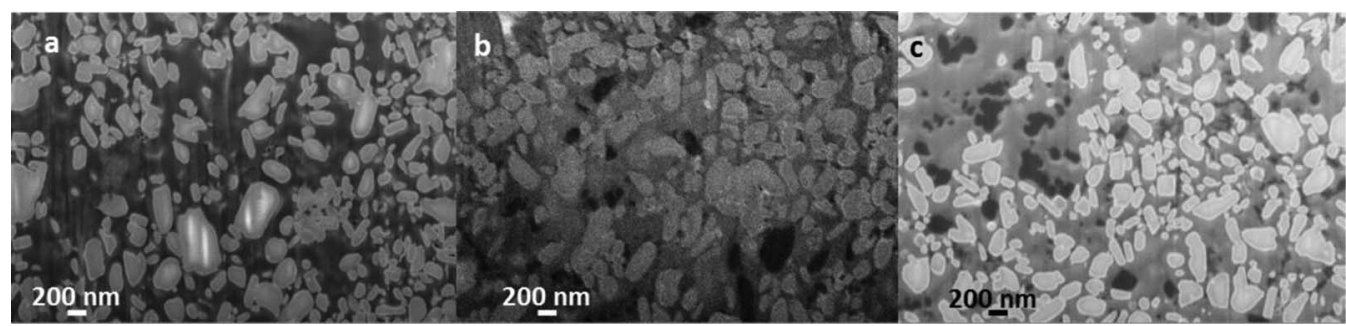

Figure 4. Cross sectional images of LFP electrode microstructure impregnated with (a) C-epoxy (b) Si-epoxy (c) Br-epoxy.

FIB-SEM tomography provides high resolution 3D images, which may reveal information otherwise not accessible with conventional lab source X-ray tomography.

The LFP sample was found to be suitable and therefore investigated using FIB-SEM tomography at high resolution, down to tens of nanometers. This resolution allows for the examination of fine particles and differentiation of the phases involved (Figure 8).

Impregnation quality.-While the C-epoxy and the Si-epoxy have already been used and found useful in terms of impregnation quality, filling most of the open pores and generating one homogenous pore phase, the Br-epoxy had not previously been reported or used, so it was important to analyze these characteristics prior to studying any contrast improvement. It is also important to note that cutting and polishing the impregnated $\mathrm{Si}$-epoxy to achieve a smooth finish is quite difficult since the Si-epoxy is relatively soft.

The Br-epoxy was found to be particularly attractive in terms of its impregnation quality as the epoxy consists of only one homogeneous and uniform phase without segregation, as shown in Figure 3. This epoxy is stable under the electron and the ion beam, and does not result in any major charging effects or related artefacts, unlike the previously used carbon and silicon based epoxies (Figures 4 and 5).

Figure 3 emphasizes the impregnation quality as the left side of Figure 3 a shows the LFP electrode impregnated with Br-epoxy, while the right side of Figure 3a shows the epoxy itself which can be seen to be dense and highly homogenous at the given resolution. The blue rectangle in Figure 3a is shown in higher magnification in Figure 3b but not yet sufficiently magnified to distinguish individual particles.

Charging and artefacts.-A common problem with imaging is that artefacts manifest during the 'slice and view' process, ${ }^{24}$ especially with porous materials due to the difference in density of the phases; uniform milling of the observation surface is then a challenging task. Some vertical lines, which are caused by preferential milling under the ion beam, often appear on the lower half of the cutting plane. They can cause local brightness change, or introduce artefacts impacting the imaging of the microstructure of the cathode,${ }^{24}$ creating difficulties in 3D image reconstruction. In Figure 4a, these lines, called the "curtaining effect" 26 as well as bright spots caused by localized charging, are visible. Imaging of the sample impregnated with the Si resin is not an easy task; with both severe charging and drifting problems evident especially in low scan speeds, resulting in a distorted image (Figure 5a). Small image drift is an unavoidable problem during image acquisition but a severe drift can adversely impact the image alignment essential for the quantitative analysis of microstructures. ${ }^{22}$ High scan speeds result in a low image quality (Figure $4 b$ ), but are needed when severe drifting is otherwise observed at low scan speeds (Figure 5a).

Coating the sample with Osmium was then carried out before beginning the milling and imaging sequence to minimize the charging and drift effects. Although the re-coated sample had a better stability under the beam, the charging problem of the Si-epoxy still exists, as can be seen in Figures $5 \mathrm{~b}$ and $5 \mathrm{c}$.

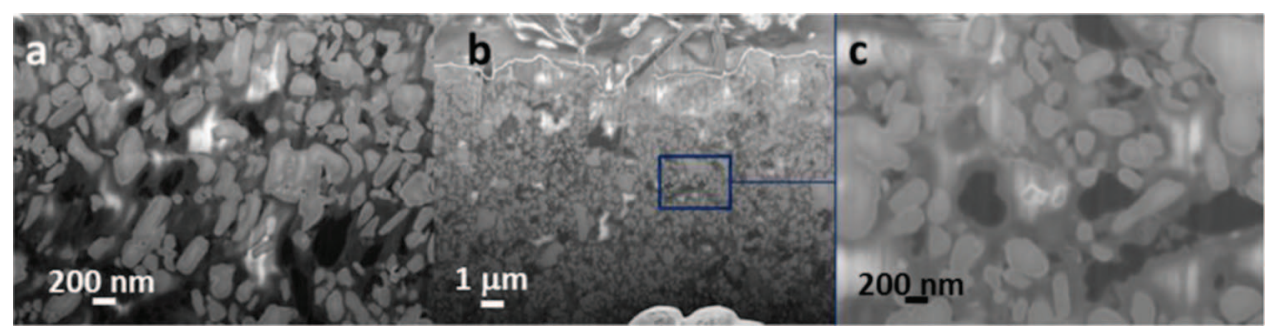

Figure 5. SEM Cross sectional images of LFP electrode impregnated with Si epoxy (a) low scan speed caused drifting (b) additional osmium coating to prevent drift in (a), and (c) low scan speed image showing a higher magnification image of the blue rectangle in (b). 

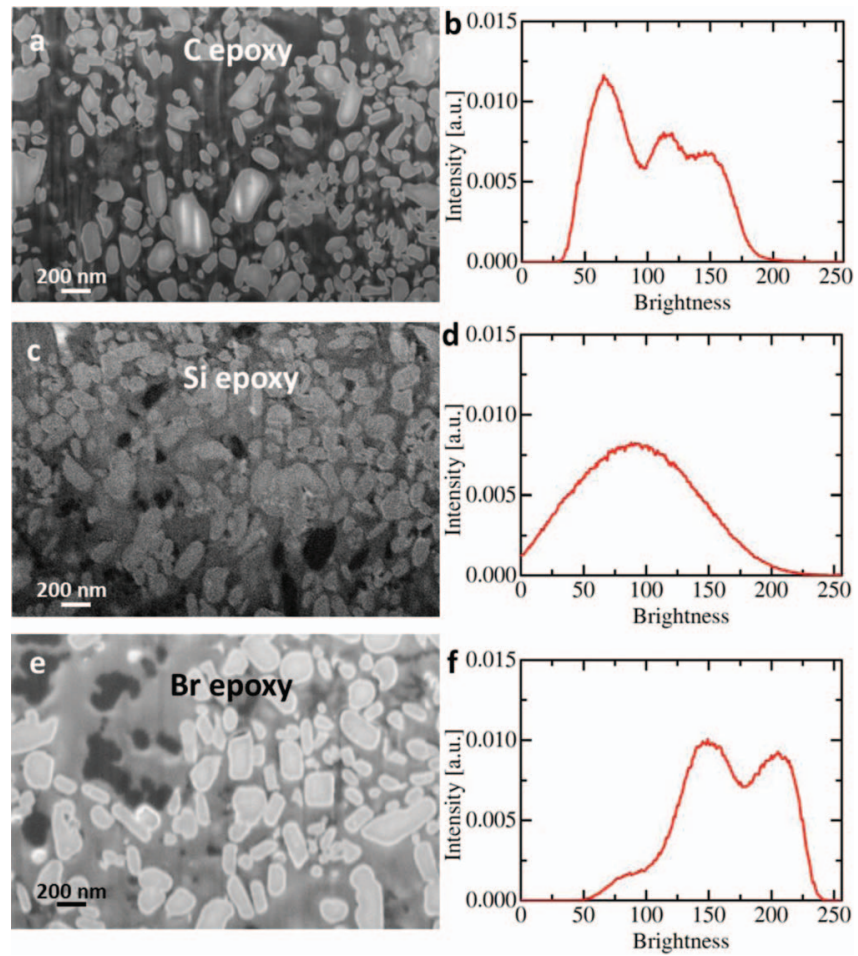

Figure 6. (a) High resolution SEM image of the LFP impregnated with Cepoxy (b) a graphical representation of the distribution of the pixel intensity values (histogram) of the left image (a). (c) High resolution SEM image for LFP impregnated with Si-epoxy, (d) histogram of (c). (e) High resolution SEM image of LFP impregnated with Br-epoxy, (f) histogram of (e).

In contrast, the Br-epoxy impregnated electrode did not show any imaging artefacts, such as drift or charging (Figure 4c). Hence, slower scan speeds were possible and higher-quality and higher-resolution images were captured. A better signal to noise is shown, therefore a better quality image in terms of sharpness and clarity is observed, which enables a fully automated segmentation process.

Contrast and ability to distinguish the different phases.-It can be clearly seen from Figure 4a that it is difficult to distinguish between the carbon epoxy (pore) phase and the small carbon black particles due to lack of contrast between the carbon particles and the C-epoxy. In contrast to the $\mathrm{C}$-epoxy, the Si-epoxy (Figure $4 \mathrm{~b}$ ) appears to show a clearer contrast to the carbon black particles, but is still not sufficient to separate the phases without overlapping, resulting in a more difficult image segmentation process. In addition, a charging problem is still observed, as discussed previously.

A histogram of the distribution of the pixel intensity values for the SEM micrographs of each of the three samples is presented in Figure 6. Each pixel has a value that represents the brightness of the pixel. A peak in the histogram indicates a gray scale level that can be associated with a given phase; hence a well resolved image will contain one distinct and resolved peak in the histogram per phase of interest.

The three different phases can be clearly distinguished in the $\mathrm{Br}$ epoxy impregnated sample, and they were also confirmed by the pixel intensity histogram (Figure 6). In the histogram of the C-epoxy (Figure $6 b$ ) it is difficult to distinguish between peaks without overlapping areas, which is not favorable for segmentation in comparison to the histogram of the Br-epoxy (Figure 4f). In the histogram of the Siepoxy (Figure 6d) only one broad peak is shown without indication of the presence of individual peaks that are overlapped across the greyscale. As a result the automated segmentation of the image is extremely difficult. However, the histogram of Br-epoxy shows 3 different brightness regions and two distinct peaks, which enable much improved image processing and segmentation, a precursor for high resolution 3D imaging and quantification. The carbon black particles which represent the lowest brightness region are only present in a small amount in the analyzed sample; hence the intensity from this region is relatively lower. Notably, the PVdF binder is likely to be an ultra-thin layer below the resolution; hence it cannot be distinguished using these techniques.
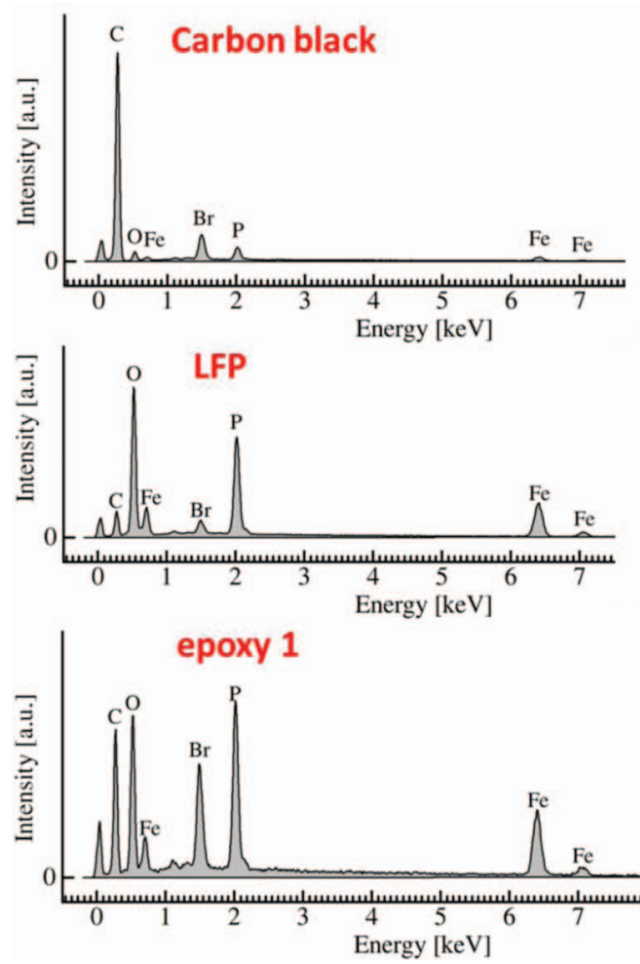

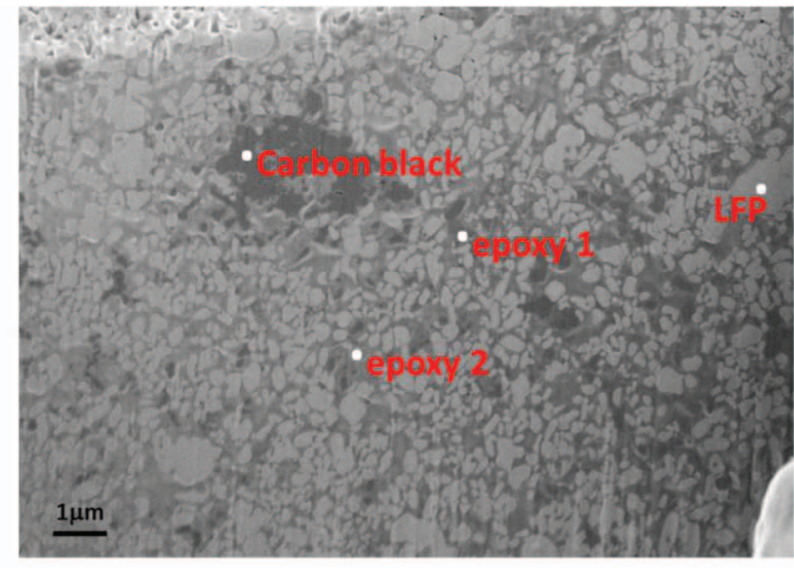

epoxy 2

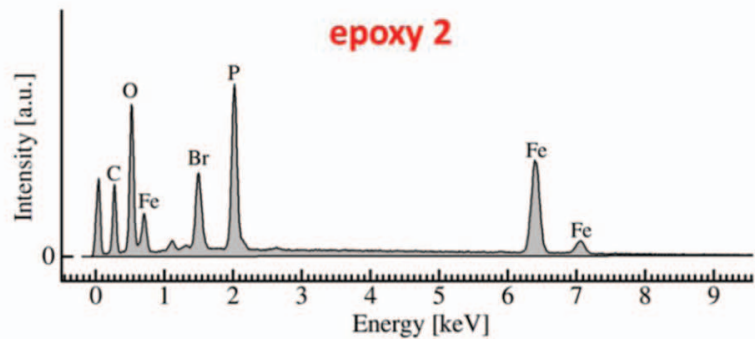

Figure 7. EDX analysis of LFP electrode impregnated with Br epoxy showing the presence of $\mathrm{Br}, \mathrm{C}$ black particles and LFP active material particles. 


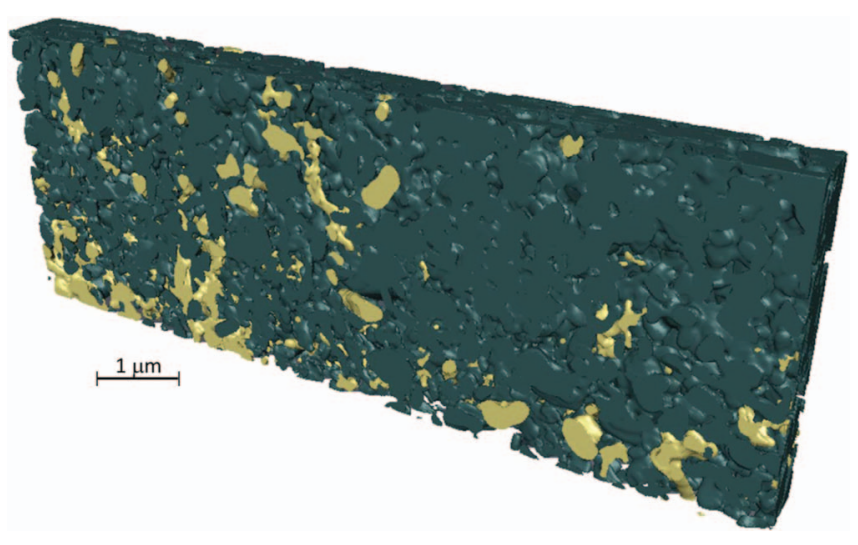

Figure 8. High resolution $3 \mathrm{D}$ image with $15 \mathrm{~nm}$ spacing and $10 \mathrm{~nm}$ pixel size $(10 \times 10 \times 15 \mathrm{~nm}-$ Blue - LFP phase, yellow - carbon black phase $)$.

The Br-epoxy appeared to be the best epoxy among the three investigated in this study in terms of image quality (Figure 6e), contrast enhancement without large areas of brightness overlapping (Figure 6f) and impregnation quality (Figure 3).

Energy dispersive X-ray (EDX) spectroscopy inspection (Figure 7) confirms that the dark particles are carbon particles. Although $\mathrm{P}$ and $\mathrm{Fe}$ were indicated in the gray phase, $\mathrm{Br}$ intensity is significantly higher in this phase than other areas, which confirms the gray area is the epoxy/pore. Strong P and Fe peaks are observed in the brightest areas, which represent the LFP particle. Since Li is a light element and the $\mathrm{X}$-ray fluorescence yield probability is very low, it was undetectable using the EDX analysis. The work shows that the Br-epoxy has the ability to distinguish between different phases for LFP composite cathode.

Advanced quantification and significance of results.-A fully automated high resolution 3D image segmentation of 50 cross-section images was applied. Importantly, no time consuming manual segmentation was required due to the high quality contrast images. This was shown to be an issue in previous studies as the carbon black and epoxy-filled pores had similar contrast. ${ }^{15}$ This also eliminates any human error or unconscious bias in the data analysis.

To the authors knowledge the resulting reconstructed 3D image (Figure 8) represents the highest resolution 3D image reported for LFP electrode materials (ca. $10 \mathrm{~nm}$ lateral $(\mathrm{x}-\mathrm{y})$ spatial resolution and $15 \mathrm{~nm}$ longitudinal (z) spatial resolution), with the ability to image tens of nanometer-sized particles.

High resolution imaging often reduces the field of view acquired for a given sample. In this study a $10 \mathrm{~nm}$ lateral and $15 \mathrm{~nm} \mathrm{z}$-depth voxel size was achieved, with an average of 20 LFP particles resolved in the $\mathrm{x}, \mathrm{y}$, and 5 in the $\mathrm{z}$, direction. It is generally accepted that at least 7-10 particles along a given axis need to be analyzed for the sample to be taken as being volume representative. ${ }^{27}$ Hence in this work, with an average particle size of carbon and LFP of $<200 \mathrm{~nm}$, and field of view of $10 \mu \mathrm{m} \times 4 \mu \mathrm{m} \times 1 \mu \mathrm{m}$, the imaged volume can be considered

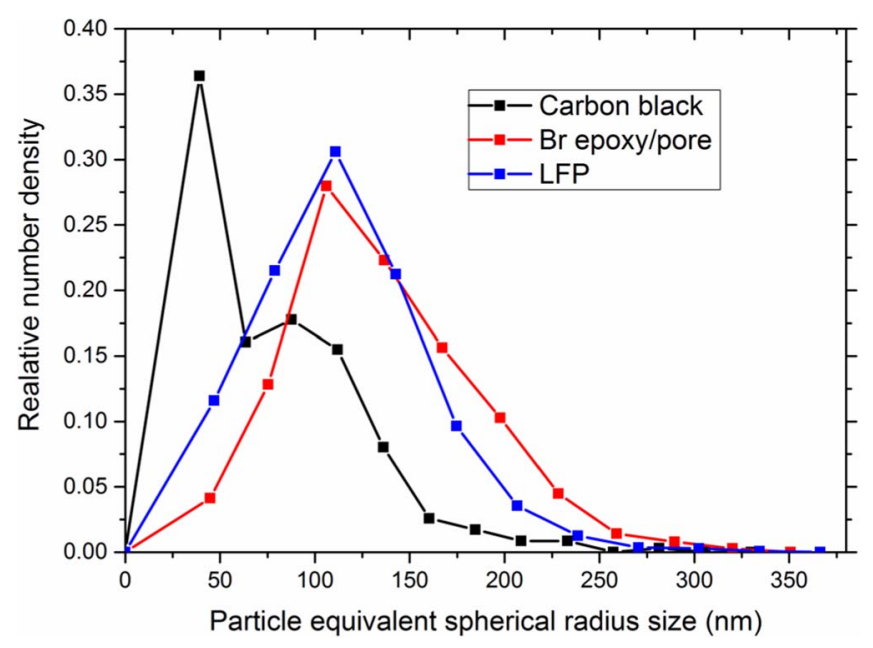

Figure 10. (black) Carbon particle size distributions, (blue) LFP particle size distribution and (red) a pore size distribution.

representative of the bulk electrode in the $\mathrm{x}$ and $\mathrm{y}$ directions, but the data quantified in the $\mathrm{z}$ direction may not be fully representative.

In a previous study there was an attempt to quantify various phases with particle size distribution. ${ }^{2}$ However, given the excellent imaging quality attained here, it was possible to apply algorithms for particle separation and advanced quantification to break down the complex 3D structure of the LFP phase (Figure 9a) and the carbon phase (Figure 9b) using proprietary software QuIQ 3D (IQM Elements, London, UK) a development of the utilities by ITK Kitware (Kitware Inc, New York, USA) methodology ${ }^{28}$ described elsewhere. ${ }^{29,30}$ In doing so, particle sizes, shapes, distributions as well as their interfaces, and the number and nature of neighboring particles were quantified. The size of the particles of each phase is shown in Figure 10. It can be seen that the particle size distribution of the carbon black particles is not uniform, which potentially may cause localized performance loss and degradation of such electrodes. ${ }^{31}$ Furthermore the spatial distribution of the carbon black phase in the reconstructed volume is also not uniform (Figure 9b). Dominko et al. have reported on the role of the carbon black distribution in cathodes for $\mathrm{Li}$ ion batteries and indicated that the uniformity of carbon black distribution significantly affects the cathode kinetics. ${ }^{31}$

As the uniformity of carbon black distribution is reported to be important, the interfaces between the carbon black particles and the LFP particles becomes of particular significance, since the role of the carbon black phase is to provide electronic conductivity. Figure 12 visualizes for the first time, to the knowledge of the authors, the interfaces between two important phases, namely carbon black and LFP, which affect the electrode kinetics.

The roundness graph (Figure 11) presents particle equivalent spherical radius size versus average of roundness for carbon particles and LFP particles (black and red respectively). It is based on a hyper sphere fit around a given particle and its subsequent deviation
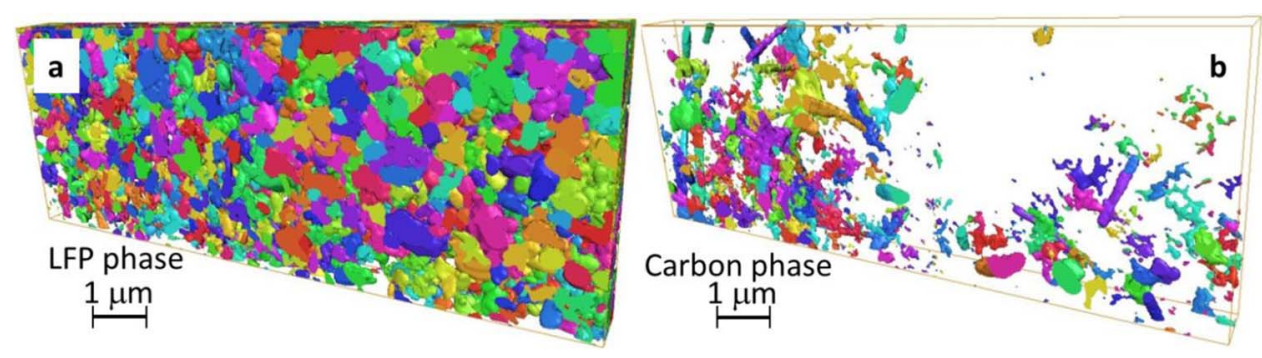

Figure 9. High resolution 3D image of (a) LFP particle size distribution (b) Carbon particle size distribution (each color represent a different particle size which is quantified and described in Figure 10). 


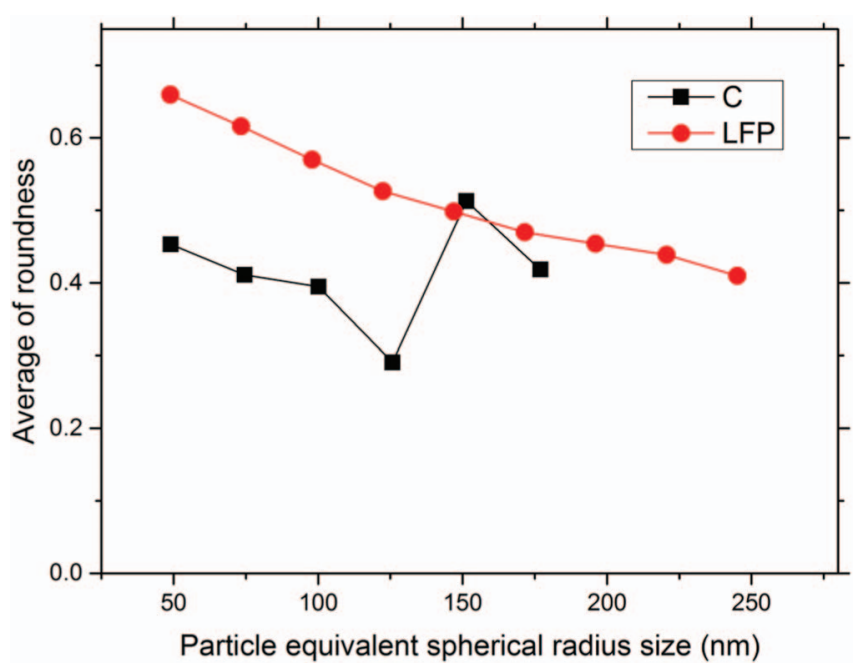

Figure 11. Particle equivalent spherical radius size versus average of roundness for (black) carbon particles, and (red) LFP particles.

from it. A perfect sphere would have a roundness value of 1 and irregular shaped particles would have lower values. ${ }^{32}$ As seen from Figures 9 and 11, the carbon black particles are less rounded than the LFP particles across most sizes for which there is data. In addition, as particle sizes increase, roundness is found to decrease for both phases. However, for carbon particles $>125 \mathrm{~nm}$ equivalent spherical radial size an increase in roundness is observed, which maybe caused through carbon particle agglomeration.

Moreover, useful metrics were calculated to present the number of neighbors and the interfacial equivalent spherical radius of the interfaces. These graphs emphasize how these small particles of tens of nanometer are structured, and how they can potentially impact on electrode performance. That result also provides a basis for tracking degradation mechanisms in LFP and other electrodes, by comparing the interfaces within a fresh and cycled electrode.

According to the graphs in Figures 10, 12b and 12c it can be seen that most of the carbon black particles have a size $<100 \mathrm{~nm}$, and almost all of them are in a good contact with the LFP phases (Figure $12 b, 12 c)$ which means that the surfaces of those particles are well utilized for electrochemical reaction.

Following the contrast improvement and the quantifiable values from the graphs (Figure 10,11, 12b and 12c), a more accurate 3D data set is obtained, which offers an excellent basis for mechanical and electrochemical property/performance simulations, relating electrode structure with performance which is an important area of development in electrochemical devices more generally. ${ }^{33,34}$

\section{Conclusions}

A novel Br-epoxy was successfully developed and applied for contrast improvement when imaging lithium ion battery carbon based electrodes. This delivered superior contrast and fewer imaging artefacts than other previously reported epoxies, enabling a full automated segmentation process. Use of the Br-epoxy made it possible to quantify the particles size and distribution within the battery electrode. This confirmed that the size and distribution of the carbon black particles
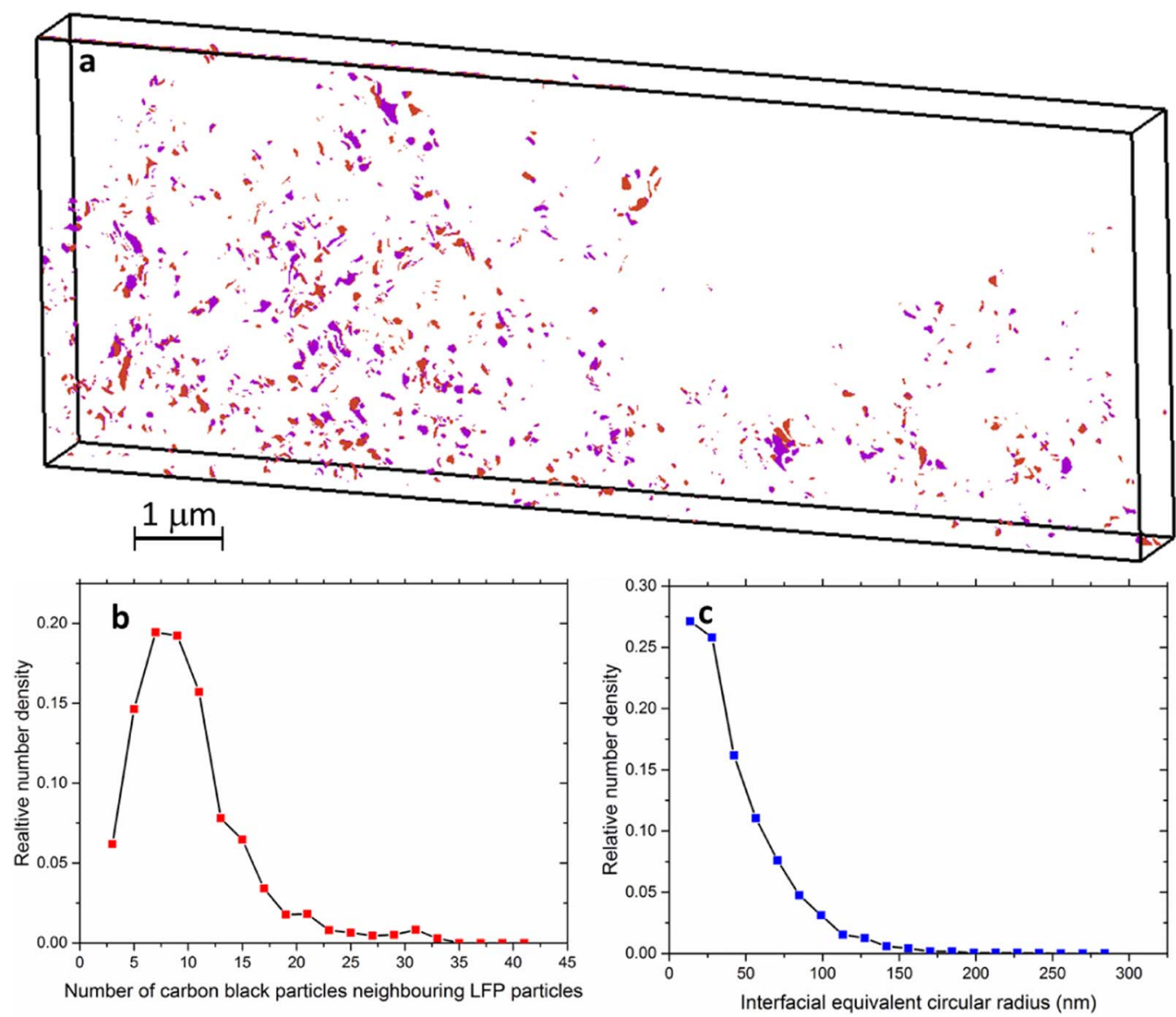

Figure 12. (a) 3D image of the interfaces between the LFP phase and the Carbon black phase, statistics from this image can be seen in (b) the number of neighbors and (c) normalized number of interfaces with an interfacial equivalent radius. 
were non-uniform; these inhomogeneities are likely to affect performance and provide cause for initiating degradation within battery electrodes.

The Br-epoxy worked very well with the carbon materials studied here, enabling high resolution (10 $\mathrm{nm}$ lateral ( $\mathrm{x}-\mathrm{y})$ spatial resolution) and high quality $3 \mathrm{D}$ imaging and quantification that could also be applied more widely to a greater range of materials. In summary, this method offers a simple and effective route for high quality 3D imaging, compared to the other methods previously reported.

\section{Acknowledgment}

We acknowledge the EPSRC (EP/L019469/1) for support. Moshiel Biton thank The STFC Network in Batteries and Electrochemical Energy Devices and the Alan Howard scholarship for further financial support (609201). We would also like to thank the MARS-EV project (FP7-2013-GC-Materials). We would also like to thank Hydro Quebec and particularly Dr Karim Zaghib for providing the electrodes for this project and Prof. Koichi Eguchi and Kyoto University that provided access to the FIB-SEM facility. Also thank IQM Elements for the evaluation software for advanced quantification.

\section{References}

1. F. Tariq, V. Yufit, M. Kishimoto, P. R. Shearing, S. Menkin, D. Golodnitsky, J. Gelb, E. Peled, and N. P. Brandon, J. Power Sources, 248, 1014 (2014).

2. M. Ebner, F. Geldmacher, F. Marone, M. Stampanoni, and V. Wood, Advanced Energy Materials, 3, 845 (2013).

3. P. Shearing, D. Brett, and N. Brandon, International Materials Reviews, 55, 347 (2010).

4. P. Shearing, L. Howard, P. S. Jørgensen, N. Brandon, and S. Harris, Electrochemistry communications, 12, 374 (2010).

5. P. Shearing, N. Brandon, J. Gelb, R. Bradley, P. Withers, A. Marquis, S. Cooper, and S. Harris, Journal of The Electrochemical Society, 159, A1023 (2012).

6. R. E. García and Y.-M. Chiang, J. Electrochem. Soc., 154, A856 (2007).

7. O. K. Park, Y. Cho, S. Lee, H.-C. Yoo, H.-K. Song, and J. Cho, Energy \& Environmental Science, 4, 1621 (2011)

8. S.-Y. Chung, J. T. Bloking, and Y.-M. Chiang, Nature materials, 1, 123 (2002).

9. B. Scrosati and J. Garche, Journal of Power Sources, 195, 2419 (2010).
10. A. K. Padhi, K. Nanjundaswamy, and J. Goodenough, J. Electrochem. Soc., 144, 1188 (1997).

11. N. Ravet, Y. Chouinard, J. Magnan, S. Besner, M. Gauthier, and M. Armand, J. Power Sources, 97, 503 (2001).

12. M. Ender, J. Joos, T. Carraro, and E. Ivers-Tiffée, Electrochem. Commun., 13, 166 (2011).

13. K. Zaghib, A. Mauger, H. Groult, J. B. Goodenough, and C. M. Julien, Materials, 6, 1028 (2013).

14. M. Biton, F. Tariq, V. Yufit, M. Kishimoto, P. Shearing, S. Cooper, D. Golodnitsky, E. Peled, and N. Brandon, Three Dimensional Microstructural Characterization of Lithium Ion Battery Electrodes, in 2014 MRS spring meeting and exhibit, in 2014 MRS spring meeting and exhibit, San Fransisco (2014).

15. M. Ender, J. Joos, T. Carraro, and E. Ivers-Tiffée, Journal of The Electrochemical Society, 159, A972 (2012).

16. S. Cooper, D. Eastwood, J. Gelb, G. Damblanc, D. Brett, R. Bradley, P. Withers, P. Lee, A. Marquis, and N. Brandon, Journal of Power Sources (2013).

17. S. A. Channagiri, S. C. Nagpure, S. Babu, G. J. Noble, and R. T. Hart, J. Power Sources, 243, 750 (2013).

18. S. Vierrath, F. Güder, A. Menzel, M. Hagner, R. Zengerle, M. Zacharias, and S. Thiele, J. Power Sources, 285, 413 (2015)

19. L. M. Keller, P. Schuetz, R. Erni, M. D. Rossell, F. Lucas, P. Gasser, and L. Holzer, Microporous Mesoporous Mater., 170, 83 (2013).

20. L. Reimer, Springer series in optical sciences, 45, ALL (1998).

21. S. Ghosh, H. Ohashi, H. Tabata, Y. Hashimasa, and T. Yamaguchi, Int. J. Hydrogen Energy, 40, 15663 (2015).

22. H. Iwai, N. Shikazono, T. Matsui, H. Teshima, M. Kishimoto, R. Kishida, D. Hayashi, K. Matsuzaki, D. Kanno, and M. Saito, J. Power Sources, 195, 955 (2010).

23. M. Kishimoto, H. Iwai, M. Saito, and H. Yoshida, J. Power Sources, 196, 4555 (2011).

24. M. Kishimoto, H. Iwai, M. Saito, and H. Yoshida, ECS Transactions, 25, 1887 (2009).

25. Sigma-Aldrich, in (2016).

26. N. Vivet, S. Chupin, E. Estrade, T. Piquero, P. Pommier, D. Rochais, and E. Bruneton, J. Power Sources, 196, 7541 (2011).

27. J. Joos, M. Ender, T. Carraro, A. Weber, and E. Ivers-Tiffée, Electrochimica Acta, 82, 268 (2012).

28. L. Ibanes, W. Schroeder, and L. Ng, The ITK Software Guide, 1st Ed., Kitware Inc., New York, NY (2003).

29. F. Tariq, M. Kishimoto, V. Yufit, G. Cui, M. Somalu, and N. Brandon, J. Eur. Ceram. Soc., 34, 3755 (2014).

30. R. Beare and G. Lehmann, Insight J (2006).

31. R. Dominko, M. Gaberscek, J. Drofenik, M. Bele, S. Pejovnik, and J. Jamnik, J. Power Sources, 119, 770 (2003).

32. G. Lehmann, Insight J, $\mathbf{8}$ (2007).

33. F. Tariq, M. Kishimoto, S. J. Cooper, P. R. Shearing, and N. P. Brandon, ECS Transactions, 57, 2553 (2013).

34. M. Kishimoto, M. Lomberg, E. Ruiz-Trejo, and N. P. Brandon, J. Power Sources, 266, 291 (2014). 